\title{
Contraste entre la práctica y las creencias epistemológicas sobre las matemáticas, su enseñanza y aprendizaje. Un estudio de casos con docentes de matemáticas en formación
}

\author{
Contrast between the practice and epistemological beliefs about \\ mathematics, its teaching, and learning. \\ A case study with pre service mathematics teachers
}

\author{
Grace Judith Vesga-Bravo ${ }^{*}$ \\ ORCID iD 0000-0002-9990-360X \\ Zaida Mabel Angel-Cuervo ${ }^{2 * *}$ \\ ORCID iD 0000-0002-7149-6336
}

\begin{abstract}
Resumen
En este artículo se presenta el resultado de una investigación cuyo objetivo fue analizar y describir las creencias epistemológicas de dos docentes de matemáticas en formación sobre las matemáticas, su enseñanza y aprendizaje y su coherencia con la práctica docente. Se utilizó un diseño de casos múltiples. Como instrumentos se usaron cuestionarios cerrados, entrevistas semiestructuradas, planes y observaciones de clases. Los resultados obtenidos indican que, no necesariamente, las creencias declaradas por los docentes se articulan, de manera coherente, con la práctica. Además aportan información que permite que los programas de formación inicial y continua revisen sus procesos curriculares, provean más y varias experiencias de aprendizaje que permita a los docentes reflexionar, de manera explícita, sobre sus creencias, su impacto en el ejercicio docente y su articulación con orientaciones curriculares actuales.
\end{abstract}

Palabras clave: Creencias del profesor. Enseñanza de las matemáticas. Formación de formadores. Práctica docente. Epistemología.

\begin{abstract}
This research aimed at analyzing and describing the epistemological beliefs that two pre-service mathematics teachers have concerning mathematics and mathematics teaching and learning as well as its alignment to the teaching practicum. A multiple-case design was used, including closed-ended questionnaires, semi-structured interviews, lesson plans, and classroom observations. The obtained results indicated that the declared beliefs are not necessarily articulated in a coherent way with the practice. These results provide information for initial and inservice training programs to review their curricular processes and design various learning experiences that allow teachers to explicitly reflect on their beliefs, their impact on the teaching practice, and their articulation with current curricular orientations.

1* Doctora en Educación Matemática de la Universidad Antonio Nariño (UAN). Docente Asociado de la Facultad de Educación de la Universidad Antonio Nariño (UAN), Bogotá, Cundinamarca, Colombia. E-mail: gvesga@uan.edu.co.

2** Magister en Educación de Universidad Pedagògica Nacional (UPN). Docente de la Facultad de Educación de la Universidad Antonio Nariño (UAN), Bogotá, Cundinamarca, Colombia. E-mail. E-mail: zaidaangel@uan.edu.co.
\end{abstract}


Keywords: Teacher's beliefs. Mathematics teaching. Training of trainers. Teaching practice. Epistemology.

\section{Introducción}

En el campo de la educación matemática se reconoce que las concepciones que tienen los docentes sobre la naturaleza de las matemáticas, así como concepciones de tipo psicológico y pedagógico influyen en su enseñanza. Esto ha hecho que se investigue sobre lo que el profesor piensa, sabe o hace (FONT, 2003; FONT; RAMOS, 2005).

Ubicados en el paradigma del pensamiento del profesor, varios investigadores se han interesado en analizar y describir las creencias que tienen sobre las matemáticas, su enseñanza y aprendizaje docentes en formación y en ejercicio, y en algunos, casos la relación de dichas creencias con la práctica docente, con las creencias de sus propios estudiantes y con la solución de problemas, y con creencias de los mismos docentes sobre su autoeficacia, entre otros aspectos (PAJARES, 1992; CHRYSOSTOMOU; PHILIPPOU, 2010; ANDREWS; XENOFONTOS, 2015; CROSS, 2009, 2015; VESGA; FALK, 2016, VESGA; DE LOSADA, 2018; BERK; CAI, 2019; CONNER; GOMEZ, 2019; YANG et al., 2020; ALBANESE; PERALES, 2020; MCMINN; ALDRIDGE; HENDERSON, 2020).

Los primeros estudios en este campo los inició Thompson (1984), quien evidenció la importancia que tienen las creencias de los docentes en las decisiones que toman frente a su práctica, y la compleja relación que existe en el sistema de creencias. Las creencias epistemológicas son creencias personales sobre el conocimiento y la adquisición del conocimiento, y conocer las que tienen los docentes es útil para comprender e, incluso, predecir las decisiones que toman (ERNEST, 1989; HOFER; PINTRICH, 1997).

En la literatura existen varias maneras de interpretar las creencias sobre la naturaleza de las matemáticas. Una de estas considera que al existir múltiples filosofías de las matemáticas que incluyen entre otras, intuicionismo, logicismo, formalismo, platonismo y empirismo, se pueden agrupar en dos puntos de vista amplios: el absolutista y falibilista (LERMAN, 1990). En correspondencia, las creencias sobre la enseñanza y aprendizaje de las matemáticas se pueden agrupar en tradicionalista y constructivista respectivamente (COONEY; SHEALY; YARVOLD, 1998; PENN, 2012; VESGA; FALK, 2016; VESGA; DE LOSADA, 2018). Estas posturas se utilizaron para el desarrollo de esta investigación, siendo conscientes de que no es una distinción binaria, y que no hay un acuerdo en la comunidad académica. Pero hay una gradación.

En el absolutismo se considera que la matemática es una ciencia formal y exacta, donde 
no hay lugar para el error; las verdades son universales e incuestionables (ERNEST, 1991, 1998). El conocimiento matemático es un cuerpo que contiene verdades absolutas, ciertas e indiscutibles (XENOFONTOS, 2018) y proviene de fuentes formales (SCHOMMER, 1990; PENN, 2012).

Se utiliza un lenguaje formal, sin errores ni contradicciones (WHITE-FREDETTE, 2009). Las matemáticas existen en un mundo de ideas puras, o en la mente del creador, y se descubren a través de sistemas lógico deductivos (ERNEST, 1991, 1998). Desde esta postura, la matemática es una disciplina aislada y discreta, claramente separada y demarcada de otros ámbitos del conocimiento (SCHOMMER, 1990; ERNEST, 1991).

En contraste, en el falibilismo se considera que la matemática es una creación de la mente humana, y se desarrolla a través de pruebas y refutaciones. Las conjeturas se van revisando y refinando según los hechos. No se trata, simplemente, de un aumento constante y monótono de verdades indubitables (LAKATOS, 1976; DAVIS; HERSH; MARCHISOTTO, 2012). Los objetos matemáticos, al ser creados por la mente humana, son imaginarios, existen primero en la mente del matemático y, luego, ganan vida propia más allá del individuo. En este sentido, las matemáticas se preocupan por la verdad de los datos de los objetos imaginados (SKOTT, 2004).

El conocimiento es falible y corregible, se construye por una comunidad que comparte significados y el proceso para obtener nuevo conocimiento puede ser, incluso, más importante que el producto (PHILLIPS, 2000; PENN 2012). Los conceptos matemáticos no están fijados de manera permanente y pueden ser modificados a lo largo del tiempo (LAKATOS, 1976; SCHOMMER, 1990). La verdad matemática deja de ser absoluta y puede estar condicionada por el contexto, o la contingencia histórica, lo que implica, al menos en el campo de la educación matemática, repensar el papel de la demostración matemática. La aceptación de una prueba es un acto fundamentalmente social (ERNEST, 1999). Así, es importante el trabajo en solución de problemas, ya que permite desarrollar procesos matemáticos (XENOFONTOS, 2018).

En el tradicionalismo se considera que el docente es el centro del proceso de enseñanza y aprendizaje, es quien tiene el conocimiento y lo trasmite. En contraste, el estudiante es un receptor pasivo que recibe información (ERNEST, 1991). Las matemáticas son consideradas como una disciplina estática, que se construye a partir de reglas basadas en procesos de memorización (RAHIME, 2015). Los problemas son escogidos por el docente, las soluciones deben ser fácilmente obtenidas al aplicar algoritmos y son correctas o incorrectas (STEINER, 1987; BESWICK, 2012). 
El maestro considera que la matemática es un conocimiento científico y objetivo, y que se debe transmitir a los estudiantes sin tener en cuenta sus ideas. La enseñanza, por otra parte, debe realizarse de forma secuencial y una fuente de conocimiento es el libro de texto (CONTRERAS, 2009). Las matemáticas son esencialmente reglas y verdades que el docente trasmite, de manera magistral, y el papel de los estudiantes es asimilar y practicar hasta que puedan hacer lo mismo con fluidez (SWAN, 2007). Se da importancia a la memorización, y se considera que el estudiante aprende si logra reproducir la información que le han dado y lo hace a través de la práctica repetitiva y rutinaria (ERNEST, 1991; PENN, 2012).

Para el docente, los estudiantes no aportan ideas relevantes a la clase, su papel es actuar como receptores de conocimiento que van acumulando; las interacciones entre docente y estudiantes se caracterizan por preguntas que hace el profesor, cuya respuesta es cerrada. El docente es rígido y controlador, y el estudiante le debe obediencia y respeto (MANSOUR, 2009).

Como contraposición a la postura anterior, surge la postura constructivista. Esta se centra en el estudiante (RAHIME, 2015), y una característica es que se generan procesos de aprendizaje activos (BUSTOS, 2012). El aprendizaje es inherentemente creativo como resultado de construcción de conocimiento (BESWICK, 2012). El docente es un orientador que lidera procesos de asesoría, o retroalimentación, en la medida en que los estudiantes lo requieran, y considera el error como parte del proceso (PONCE, 2008).

El profesor, a partir de las ideas y conocimientos previos de sus estudiantes, selecciona y organiza los contenidos a abordar, su metodología no se basa en el uso de una única fuente, y usa diferentes materiales que faciliten la construcción de conocimiento de manera individual y colaborativa (CONTRERAS, 2009). El conocimiento, lo construyen activamente las personas a partir de sus conocimientos previos y como resultado de operaciones mentales (LEINO, 1994; BALL; BASS, 2000).

Por lo tanto, se deben crear entornos de aprendizaje que permitan el descubrimiento, el desarrollo del pensamiento crítico y el trabajo en equipo. Los estudiantes, por otro lado, deben ser investigadores activos, mientras que el docente facilita el aprendizaje planteando preguntas desafiantes, y evita el uso de recompensas y castigos (CONNER et al., 2011). El lenguaje influye y es importante en la construcción del conocimiento. A través de éste, se justifica y comunica (ERNEST, 1991; PHILLIPS, 2000). Se considera que el estudiante logra el aprendizaje cuando puede resolver problemas tiempo después del período de instrucción y en una amplia variedad de situaciones (PEHKONEN; TÖRNER, 1999).

Actualmente, es ampliamente aceptado que las creencias que tienen los docentes y su 
práctica están estrechamente relacionadas y pueden facilitar, u obstaculizar, cambios curriculares (CROOS, 2009; CHEN; LEUNG, 2010; ZHAO; MOK; CAO, 2016). Las tendencias actuales de la educación matemática, orientadas hacia el aprendizaje constructivista, sólo pueden tener éxito si se consideran y confrontan las creencias de los profesores (HANDAL; HERRINGTON, 2003; PANTZIARA; KARAMANOU; PHILIPPOU, 2013; XIE; CAI, 2020).

Algunos estudios también han señalado la importancia de incluir el análisis de la práctica de los docentes en formación o en ejercicio cuando se quieren analizar estas creencias, porque es en la práctica donde se explicitan y pueden identificar comportamientos particulares (BESWIK, 2012; VESGA; FALK, 2016; VESGA; DE LOSADA, 2018)

De otra parte, la correspondencia entre las creencias sobre las matemáticas y su enseñanza y aprendizaje no necesariamente es una correspondencia biunívoca. Diferentes estudios han mostrado que es usual encontrar posturas falibilistas sobre la matemática y de tipo tradicional sobre su enseñanza y aprendizaje, o absolutistas y constructivistas, es decir, posturas mixtas (BESWICK, 2012; PENN, 2012; VESGA; DE LOSADA, 2018; YANG et al., 2020). Por lo descrito, el interés en esta investigación es indagar en las creencias que declaran docentes en formación y contrastarlas con las que pueden evidenciarse en su práctica de aula, y de este modo, tener información que permita identificar qué tanta coherencia existe entre las posturas que se declaran y las que efectivamente pueden observarse, así como la coincidencia o no de estas con las creencias que se promueven actualmente.

\section{Metodología}

Para este estudio se reportan las creencias de dos docentes en formación sobre las matemáticas, su enseñanza y aprendizaje y se contrastan con lo observado en su práctica docente. Específicamente, las preguntas que orientan este estudio son: ¿Cuáles son las creencias que tienen sobre la matemática, su enseñanza y aprendizaje dos docentes de matemáticas en formación? y ¿Existe coherencia entre las creencias reportadas y la práctica docente que realizan?

La investigación corresponde con un estudio de casos múltiples (YIN, 2008), que permite involucrar métodos mixtos, enmarcada dentro de una investigación confirmatoria (ROCCO et al., 2003). Con las preguntas de investigación planteadas, se logra realizar un análisis cuantitativo sobre cualitativos que se codifican. Los docentes en formación (DF) fueron seleccionados de manera intencionada, se vincularon de manera voluntaria y se identifican como Joaquín y Margarita. Los dos habían cursado más del 50\% de los créditos del programa 
de Licenciatura en Matemáticas, de una Universidad de carácter privado en Colombia, y estaban realizando, de manera simultánea, su práctica pedagógica en instituciones educativas de carácter oficial en la ciudad de Bogotá. Este fue el criterio tenido en cuenta para la selección.

\subsection{Instrumentos}

Se utilizaron cuestionarios cerrados, entrevistas semiestructuradas, planes de clase y formatos de acompañamiento in situ. Se utilizaron los cuestionarios cerrados de Vesga y Falk (2016) para determinar posturas sobre las creencias. Un cuestionario tenía 29 afirmaciones y permitía identificar posturas o tendencias de tipo absolutista o falibilista frente a las matemáticas, y el otro, con veinte afirmaciones, se usó para identificar posturas o tendencias tradicionales o constructivistas sobre su enseñanza y aprendizaje. Para cada afirmación los DF debían marcar en una escala Likert, con una graduación de 1 a 5, qué tanto estaban de acuerdo. Estos instrumentos fueron diligenciados por cada participante al inicio y al final del semestre. Por ejemplo, en el primer instrumento una de las afirmaciones era El conocimiento matemático es falible y corregible, como cualquier ciencia humana y en el segundo Cualquier persona puede aprender matemáticas.

Con base en las respuestas dadas en los instrumentos cerrados se diseñaron entrevistas semiestructuradas para indagar y profundizar sobre la justificación y el origen de las creencias señaladas. Por ejemplo, se les preguntó ¿por qué esta complemente de acuerdo (o en desacuerdo) con que el conocimiento matemático es falible y corregible como cualquier ciencia humana? Los DF respondieron las entrevistas al comienzo y al final del semestre, una semana después de haber respondido los instrumentos cerrados, con una duración promedio de diez minutos. Adicionalmente, en la primera entrevista se preguntó por la motivación para ser docente de matemáticas y lo que esperaban que sus estudiantes aprendieran; en la segunda, por algunos cambios reportados con respecto a la primera aplicación.

Se analizaron cinco planes de clase elaborados por cada DF. Estos planes de clase deben ser elaborados antes de cada intervención en el aula, son un requisito para que el DF pueda realizar su práctica. El formato establecido por la universidad tiene cuatro aspectos: caracterización, contenidos, estrategias y recursos, y desarrollo de la clase. En el primero, se presentan aspectos generales como el nombre de la institución educativa en la cual se desarrolla la práctica, el curso a la que va dirigida, la fecha y el tiempo de duración estimada. En el segundo, se establece el eje temático a desarrollar y las competencias, logros o desempeños que se pretende que los estudiantes alcancen. Estos guardan coherencia con el plan de estudios del 
colegio y con los documentos curriculares de matemáticas. El tercero presenta los recursos que se utilizarán para el desarrollo de la sesión, y se describe, grosso modo, la metodología que planea utilizar. Por último, se expone la secuenciación de la clase. Esta debe tener una etapa de motivación (introducción al tema), otra de desarrollo (profundización del tema) y una final, de cierre (evaluación).

Un cuarto instrumento fue el formato de acompañamiento in situ. Cada DF era acompañado en algunas clases por un docente de la Licenciatura. Este permanecía durante toda la clase y hacía un reporte que daba cuenta de lo programado por el DF frente a lo observado. En el formato se sistematizaba información general sobre aspectos disciplinarios, didácticos y la percepción del docente titular de la institución sobre el proceso. Los acompañamientos fueron realizados por los autores de este trabajo.

\subsection{Análisis de la información}

Con la información reportada en los cuestionarios cerrados, se determinaron las creencias o tendencias para cada participante. Con las fórmulas establecidas por Vesga y Angel (2018), para las creencias sobre la matemática se calculó el índice denominado $C M$, un valor continuo entre 0 y 1 . Entre más cercano es el valor a 1 las creencias son de tipo falibilista mientras que el valor cercano a cero significa que son creencias de tipo absolutista. En el centro de la escala, entre 0,4 y 0,6 se considera que no están claramente definidas, como se describe en la Figura 1.

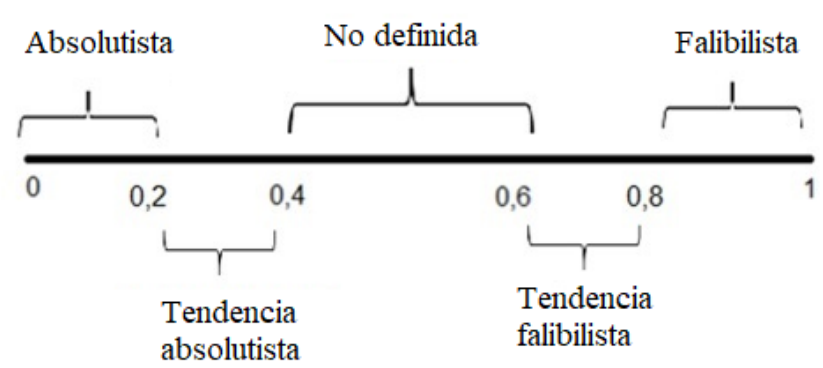

Figura 1 - Interpretación $C M$

Fuente: elaboración propia

De manera similar, se determinó un índice denominado CEAM para las creencias sobre la enseñanza y aprendizaje de las matemáticas. Se reportan los resultados del inicio y finalización del semestre, los cuales se identifican como CM1, CM2, CEAM1, CEAM2 respectivamente. Las entrevistas fueron transcritas y se usaron para analizar la coherencia con las creencias declaradas en los instrumentos cerrados. 
Los planes de clase se codificaron con uso del software Atlas ti y con uso el proceso descrito por Strauss y Corbin (2002). La codificación se hizo para identificar solo las creencias en relación con la enseñanza y aprendizaje de las matemáticas. De acuerdo con el marco teórico establecido para cada una de las categorías, constructivismo y tradicionalismo, se analizaron cinco subcategorías establecidas a priori, y confirmadas en el proceso de codificación $a$ posteriori. En el Cuadro 1 se presentan todos los códigos utilizados y la manera como se usaron durante la codificación.

\begin{tabular}{|c|c|c|}
\hline \multirow{2}{*}{ Subcategorías } & \multicolumn{2}{|c|}{ Categorías } \\
\hline & Constructivismo & Tradicionalismo \\
\hline Aprendizaje & $\begin{array}{l}\text { El conocimiento lo construyen activamente } \\
\text { las personas a partir de sus conocimientos } \\
\text { previos. }\end{array}$ & $\begin{array}{l}\text { El docente posee e imparte el } \\
\text { conocimiento. }\end{array}$ \\
\hline $\begin{array}{l}\text { Enseñanza - } \\
\text { docente }\end{array}$ & Es orientador y creativo. & Es autoritario y controlador. \\
\hline $\begin{array}{l}\text { Enseñanza - } \\
\text { estudiante }\end{array}$ & $\begin{array}{l}\text { Es activo, construye su propio } \\
\text { conocimiento, toma decisiones de forma } \\
\text { crítica. }\end{array}$ & $\begin{array}{l}\text { Es pasivo, recibe, memoriza y reproduce } \\
\text { el conocimiento. }\end{array}$ \\
\hline $\begin{array}{l}\text { Enseñanza - } \\
\text { clase }\end{array}$ & $\begin{array}{l}\text { Hay un clima dialógico, científico y } \\
\text { democrático. }\end{array}$ & $\begin{array}{l}\text { Método expositivo, no hay experiencias } \\
\text { vivenciales. }\end{array}$ \\
\hline Evaluación & $\begin{array}{l}\text { Se privilegia el papel activo del estudiante } \\
\text { como creador de su constante evolución } \\
\text { progresiva de las estructuras de } \\
\text { conocimiento. }\end{array}$ & $\begin{array}{l}\text { Hay conocimiento si el estudiante } \\
\text { memoriza conceptos y repite } \\
\text { procedimientos. Respuestas correctas o } \\
\text { incorrectas. }\end{array}$ \\
\hline
\end{tabular}

Cuadro 1 - Categorías y subcategorías utilizados para codificar los planes de clase; algunos ejemplos Fuente: elaboración propia

Por ejemplo, en el primer plan de clase de Joaquín, la parte final la denomina reflexión y perspectiva, y hace varias preguntas a sus estudiantes. Una de ellas es: ¿Qué aprendiste el día de hoy? Esto evidencia que el DF considera que el estudiante es autónomo en su aprendizaje y que está en la capacidad de autoevaluar lo aprendido de forma crítica. Este fragmento se codifica como constructivista en la subcategoría de evaluación.

Con los resultados de la codificación, se calcula el porcentaje de las afirmaciones asociadas a categoría y subcategoría. Este valor se transforma a una escala entre 0 y 1 para determinar las creencias sobre la enseñanza y aprendizaje de las matemáticas reflejadas en los planes de clase, que se denomina CEAM3 y que se interpreta de manera similar a CEAM1 y CEAM2, descritos anteriormente. Finalmente, los formatos de acompañamiento in situ se usan para dar cuenta del desarrollo de los planes de clase, para describir el papel del DF y los estudiantes, de modo que provea evidencia que permita establecer coherencia entre lo declarado a través del plan de clase y lo ejecutado y entre lo declarado en los instrumentos cerrados y la entrevista y su ejercicio como docente en formación.

\section{Resultados}


Para cada DF se describen sus posturas a partir de los instrumentos cerrados al inicio y al final del semestre. Con base en las respuestas dadas a los instrumentos, se realizaron entrevistas semiestructuradas para analizar la argumentación de las creencias. La coherencia de su práctica con las posturas señaladas se analiza con los cuatro instrumentos utilizados. Se hace un análisis que combina los resultados cuantitativos con lo cualitativo de modo que se pueda profundizar en los dos casos estudiados.

\subsection{El caso Joaquín}

Joaquín dijo que quiere ser profesor de matemáticas porque cree que a partir de su ejercicio docente puede enseñar de una forma diferente, pero, sobre todo, porque considera que puede motivar a sus estudiantes a aprender, en cierto modo a vencer el miedo y arriesgarse, intentarlo:

Quiero cambiar la idea, eh, de enseñar así, [...] quiero eh, definitivamente generar, construir conocimiento, quiero enseñarles a que se pueden arriesgar porque a partir de eso se puede aprender, si tú no arriesgas eh, el, el peor de los riesgos es no arriesgarse es eso (Entrevista No. 1, Joaquín, 2018).

También dice que es necesario enseñar de forma diferente a la tradicional, la cual asocia con repetición de procedimientos. Para Joaquín es importante que sus estudiantes le tomen cariño a las matemáticas, que cada clase sea una experiencia positiva:

Que cada día se llenen de alguna experiencia, que no le cojan, eh, esa fobia ni nada de eso, que cada vez que lleguen eh, estén listos y estén eh, felices a ver qué tema, que, qué tema y dispuestos para, para aprender (Entrevista No. 1, Joaquín, 2018).

\subsubsection{Creencias epistemológicas acerca de la matemática}

Con base en los instrumentos cerrados se pudo observar que Joaquín no tiene una postura claramente definida sobre sus creencias acerca de las matemáticas, pero se inclina hacia el falibilismo. En las dos aplicaciones se obtuvo como resultado $C M 1=0,58=C M 2$. Durante las entrevistas se pudo observar que le era difícil argumentar sus creencias. En general, las justificaciones que dio para sus creencias estaban referidas a la matemática escolar. Por ejemplo, en el instrumento cerrado dijo estar de acuerdo con que la matemática ha evolucionado a través de la historia. Durante la entrevista, al respecto señaló que fue muy importante la creación de la matemática griega y señala que ese fue un punto clave para el avance de las matemáticas: 
Desde la época de Euclides, que eso prácticamente me pareció algo muy importante, muy influyente y que me ha aportado grandes herramientas el de los cinco postulados de Euclides, los cinco postulados de Euclides y la geometría que él invento porque desde ahi se fue creando un pensamiento, sí, y desde ahi se fue, perfeccionando, a partir de ese pensamiento se fue perfeccionando y sacando nuevas herramientas y a partir de ahí, se, amplió más conocimiento (Entrevista No. 1, Joaquín, 2018).

Al mismo tiempo, señaló en el instrumento cerrado que no estaba de acuerdo con que se inventa nueva matemática cada día. En la entrevista dijo que cree eso porque siempre se enseña lo mismo. Joaquín también considera que hay varias formas de resolver un problema y eso hace parte de la construcción de nuevo conocimiento, todo depende de la creatividad del resolutor y de las bases que tenga sobre el tema que está abordando. Sin embargo, en su justificación durante la entrevista hace referencia a la solución de problemas en el aula.

Nosotros como maestros, eh, debemos tener en cuenta, un problema determinado no se resuelve de una sola forma, al resultado a una conclusión se puede llegar de diferentes formas, en diferentes caminos y si el niño lo entiende por otro camino es correcto, o sea el procedimiento es correcto y es una forma diferente de hacerlo, entonces debemos entender eso. O sea una construcción del conocimiento es eso, diseñar diferentes caminos hacia esa conclusión (Entrevista No. 1, Joaquín, 2018).

Joaquín afirma que esta debe ser la forma correcta en que un profesor de matemáticas aborde los problemas en la escuela. Relata que cuando era niño su profesor solo les valía el trabajo si lo hacían de la misma forma en la que les había enseñado. Aunque también señala que se puede producir tensión al no poder encontrar soluciones y por tanto, siente alivio cuando logra resolver una situación:

Porque nosotros al desarrollar, eh, un problema al pensar una forma de, de llegar a esa conclusión, de poder llegar a resolver ese problema nos genera como un alivio. ¿Sí?, eh, nos genera ese clima, ese alivio de haber terminado, o sea de haber terminado con esa presión de que como no puedo hacerlo (Entrevista No. 1, Joaquín, 2018).

Joaquín señaló, inicialmente, en el instrumento cerrado, que la matemática es una ciencia falible y corregible como otras ciencias humanas, lo cual lo argumentó desde su proceso de formación, considera que ha sido bueno poder aprender del error.

Definitivamente cuando encontramos, eh, problemas podemos, eh, corregir o mediante eso lograr enderezar el camino, por ejemplo en las pequeñas mentes que estamos formando, o, o bueno yo, yo fui una de esas, porque yo llegué aqui a la universidad prácticamente con ese deseo de enseñar (...) y a partir de los profesores que he tenido como que he conocido, como que se me ha abierto el conocimiento y, y lo he ampliado mucho mejor, y he corregido algunas cosas que definitivamente no eran (Entrevista No. 1, Joaquín, 2018).

En la segunda aplicación de los instrumentos, dijo que no estaba ni de acuerdo ni en desacuerdo con que la matemática sea falible y corregible. Su argumentación se basa en que si un problema esta mal se debe corregir:

Que puede fallar si, ni en desacuerdo ni en acuerdo. Cuando nosotros trabajamos problemas, 
varios problemas, porque es que la matemática es extensa, la matemática tiene unos panoramas diferentes, tiene por un lado problemas que tienen de una u otra forma algo de sentido y podemos llevar nuestro conocimiento, podemos empezar a construir con lo que sabemos esa solución, pero puede fallar en un momento un problema determinado entonces dependiendo también de ese fallo podemos corregir lo que estamos haciendo mal con otro tipo de propiedades (Entrevista No. 2, Joaquín, 2018).

Joaquín relaciona el hecho de construir la matemática a partir de la experiencia humana con poder construir matemática sin tener un cúmulo de conocimientos previos

[ ] no necesariamente uno tiene que ser eh, haber sido, o haber tenido harto conocimiento matemático para poder crear un problema, para poder resolver algo. Nosotros a partir de nuestras experiencias creo que podemos diseñar y lograr resolver un problema matemático, eh porque prácticamente lo vivimos día a día, lo encontramos día a día, en las matemáticas, sino que nos negamos, no las vemos, nos negamos a verlas, pero es prácticamente desde la experiencia que nos podemos dar cuenta y nos despertamos bien, estas están al lado de nosotros prácticamente (Entrevista No. 1, Joaquín, 2018).

De otra parte, Joaquín considera que la matemática es exacta, que los problemas siempre tienen solución y para poder trabajar se requieren algunas bases:

Formal y exacta, lo digo desde mi perspectiva, porque lo considero que es una asignatura y también una ciencia que no solamente necesita una buena capacidad de análisis sino que también necesita tener unas buenas bases y si no tienes esas bases o sea no es necesario tener todo el bagaje sino algunas, algunas bases [ ] (Entrevista No. 2, Joaquín, 2018).

Se observa, entonces, que Joaquín argumenta sus respuestas, la mayoría de las veces, con ejemplos de la matemática escolar.

\subsubsection{Creencias sobre la enseñanza y aprendizaje}

Joaquín mostró, a través del instrumento cerrado, una tendencia hacia el constructivismo (CEAM1 = 0,68 en la primera aplicación) la cual se fortaleció, de manera importante, al finalizar el semestre ( $C E A M 2=0,74$ en la segunda aplicación). Durante las entrevistas se pudo evidenciar que podía argumentar sobre esta postura. Joaquín considera que cualquier persona puede aprender matemáticas, dice especialmente que los niños pueden ser muy creativos $\mathrm{y}$, por tanto, es posible aprender de ellos:

Eh sí, eh, ellos prácticamente eh, son más pilos que uno, eh, sobre todo los niños de primaria porque ellos cuentan sus experiencias y a partir de esas logran crear. Yo veo que dibujan, eh, otros que dicen hay esto se puede resolver por otro, por este lado, entonces uno queda sorprendido y dice "oiga si, por qué no lo pensé o algo asi" (...) uno puede lograr a prender de ellos (Entrevista No. 1, Joaquín, 2018).

Joaquín también considera que el aprendizaje a través de problemas es muy importante y señala que así no aprendió cuando fue estudiante de educación básica y media, pero que a través de su formación universitaria ahora lo tiene muy claro y, por eso, es importante también 
que los estudiantes puedan desarrollar y probar teorías.

[ ] desde mi experiencia como estudiante en el colegio, porque prácticamente se limitaba a lo abstracto de números, números pero no me daban en sí un problema por eso es que cuando entre acá y me dieron un primer problema me dio duro por eso fue, porque a mi me enseñaron solo lo abstracto a mí nunca me enseñaron a partir de problemas ahí está la importancia irnos acercarnos a los niños, a través de los problemas para que ellos se vayan familiarizando con ese tipo de lenguaje (Entrevista No. 1, Joaquín, 2018).

Para Joaquín el error puede ser una fuente de aprendizaje. Señala la importancia que los estudiantes así se equivoquen se sientan seguros, aunque al final ratifica que es importante usar el error para mostrar a los demás lo que no deben hacer. En coherencia con su postura, está en desacuerdo con que el éxito del aprendizaje de las matemáticas esté en la repetición de procedimientos, señala que se puede partir de lo conocido para crear cosas nuevas, no simplemente para repetir

[ ] no se debe tener una forma tradicional de estar repitiendo los procesos, sino, a lo que, a lo que he dicho y he reiterado que es arriesgar, arriesgar mirar si hay otro camino a partir de otras herramientas como investigado nuevas formas ¿sí?, y a partir de lo que uno sabe de esos conocimientos previos, de eso, de esa forma, eh, en la que siempre lo ha desarrollado puede generar otro camino (Entrevista No. 1, Joaquín, 2018).

Finalmente para Joaquín, el docente no es el responsable de trasmitir conocimiento, aunque sí debe tener más conocimiento que sus estudiantes, pero destaca la importancia de construirlo entre todos. Se ve en sus argumentos una postura hacia el constructivismo.

\subsubsection{Creencias y relación con la práctica}

Antes de iniciar su práctica, Joaquín no había tenido ninguna experiencia como docente de aula. Su primera práctica la realizó en una institución de carácter privado, con un grupo de quince estudiantes de sexto grado, con quienes trabajó fracciones y polígonos. En la segunda, estuvo en una institución de carácter público, con dos cursos de grado séptimo, aproximadamente treinta estudiantes por curso, enfocado en aspectos geométricos.

Joaquín decidió partir de una situación que le generara interés al grupo. Identificó, a través de un instrumento de caracterización del curso, que a los estudiantes les gustaba la geometría y que el fútbol era el deporte preferido por los niños y las niñas, razón por la cual seleccionó este contexto. Esto es coherente con lo señalado inicialmente sobre no enseñar siempre de manera tradicional y motivar a los estudiantes. Con base en la codificación realizada a los planes de clase se estableció que $C E A M 3=0,97$, lo que refleja una postura definida hacia el constructivismo, como se muestra en la Tabla 1.

Tabla 1 - Resultados codificación planes de clase de Joaquín 


\begin{tabular}{|l|l|c|}
\hline \multicolumn{1}{|c|}{ Categoría } & Subcategoría & Porcentaje \\
\hline \multirow{4}{*}{ Constructivismo } & Aprendizaje & 34,26 \\
\cline { 2 - 3 } & Enseñanza - clase & 19,44 \\
\cline { 2 - 3 } & Enseñanza - docente & 11,11 \\
\cline { 2 - 3 } & Enseñanza - estudiante & 23,15 \\
\cline { 2 - 3 } & Evaluación & 9,26 \\
\hline \multirow{4}{*}{ Tradicionalismo } & Aprendizaje & 0,93 \\
\cline { 2 - 3 } & Enseñanza - clase & 0,93 \\
\cline { 2 - 3 } & Enseñanza - docente & 0,93 \\
\cline { 2 - 3 } & Enseñanza - estudiante & 0,00 \\
\cline { 2 - 3 } & Evaluación & 0,00 \\
\hline
\end{tabular}

Fuente: elaboración propia

Joaquín hizo primero una evaluación diagnóstica con el objetivo de identificar las fortalezas y aspectos geométricos a trabajar, específicamente lo relacionado con polígonos, movimientos en el plano y nociones sobre el fútbol. Esto muestra que da importancia a los conocimientos previos de los estudiantes para poder programar las siguientes sesiones de clase. A partir de la información arrojada, planea la enseñanza de la geometría de grado séptimo a través del fútbol como contexto. Los temas abordados fueron las figuras y conceptos geométricos que se identifican al interior del campo de fútbol: la construcción de triángulos, medianas (en la triangulación de las jugadas), tácticas futbolísticas en el plano, rotaciones y traslaciones (en el plano de juego), y reflexiones (en la alineación del equipo).

Joaquín diseñó la secuenciación de la clase con base en la estructura de los planes de clase en cuatro momentos: i) Activación de conocimientos; ii) Compartiendo tus conocimientos; iii) Aprendiendo geometría de la mano del fútbol; y iv) Reflexión y perspectivas. Los primeros dos correspondían a la introducción y motivación hacia el tema propuesto; la tercera, al desarrollo y profundización; y la cuarta, al cierre y evaluación.

En la activación de conocimientos, Joaquín presenta una lectura sobre alguna jugada o estrategia futbolística que involucra aspectos geométricos para llevar a un equipo a una opción de gol, o a hacer jugadas que generen peligro en el área rival. Con base en esto, plantea preguntas y, luego, hace una primera socialización enlazando, así, el segundo momento. Varias de las lecturas eran adaptaciones de revistas deportivas y le permiten a Joaquín mostrar a sus estudiantes que la geometría puede ser utilizada para modelar y entender situaciones de la vida cotidiana, como el fútbol.

Por ejemplo, en el primer plan de clase presenta una descripción sobre el campo de fútbol junto con la imagen y plantea preguntas como ¿Alguna vez has pensado si la geometría está presente en el fútbol? es decir, ¿si dentro de este juego encuentras algunos elementos o 
conceptos geométricos?" (JOAQUÍN, plan de clase 1, 2018). Se observó durante el acompañamiento en aula que estos interrogantes permitieron a los estudiantes reflexionar sobre la relación de la geometría con un deporte. Joaquín promovía su participación de los estudiantes y los orientaba para que llegara a la comprensión de los conceptos previos requeridos.

Para el desarrollo del tema, aprendiendo geometría de la mano del fútbol, Joaquín profundizaba sobre el tema propuesto. En el primer plan de clases Joaquín describe una de las tácticas de juego y, a su vez, presenta, a través de la ilustración, el uso de la geometría para el diseño de estrategias. De este modo buscaba que emergieran nuevos conceptos geométricos, en este caso particular relacionados con la triangulación y traslación, como se observa en la Figura 2.

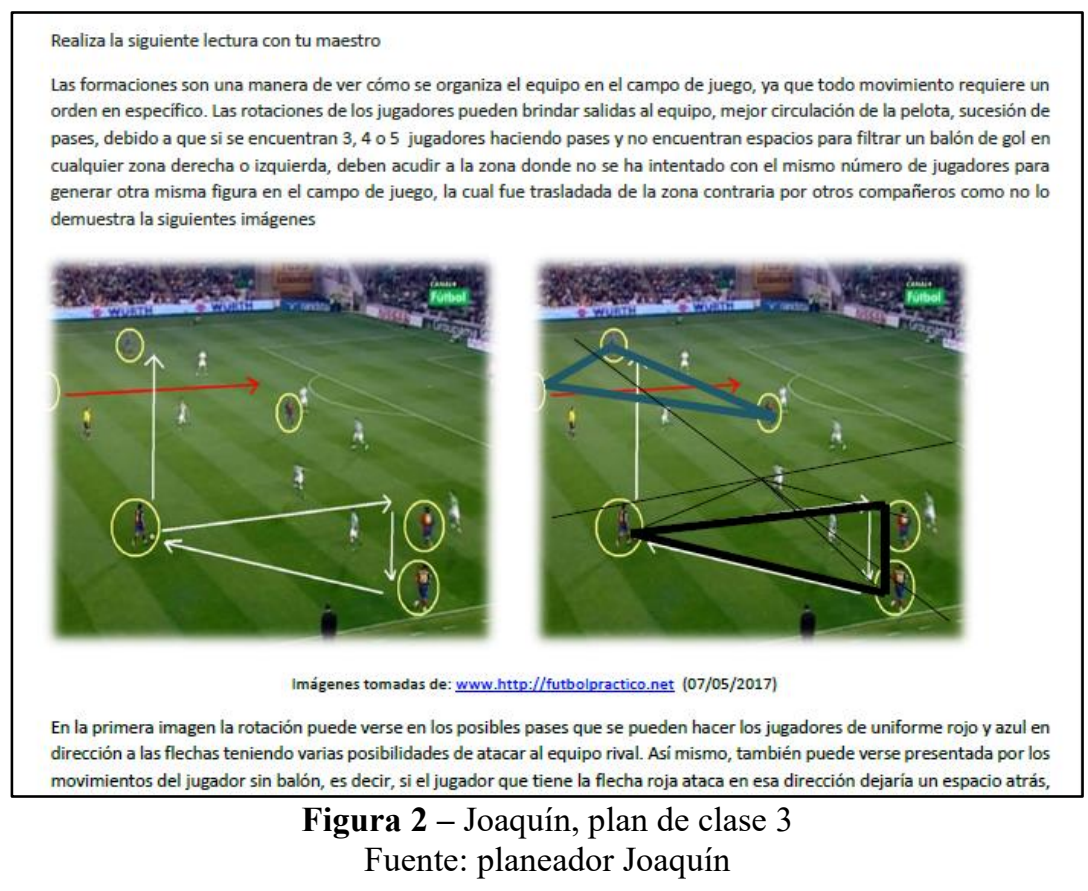

Los estudiantes señalaron en sus intervenciones: me ha hecho comprender cómo elaborar una jugada gracias a las medianas, ya que sin ellas no habría goles; esos pases que puede hacer un jugador a otro haciendo que se mueva a la mitad de la triangulación es en geometría la mediana; la mediana es el pase que va a la mitad del triángulo formado por los jugadores haciendo que se hagan jugadas de peligro y así ganar; el jugador que está en el centro del triángulo puede hacer más pases porque tiene más medianas. Estas respuestas permiten inferir que los estudiantes construyen el concepto de mediana a partir de las diferentes jugadas futbolísticas. Si bien ellos no establecen la definición formal de la matemática escolar, Joaquín, a partir de sus aportes, la formaliza de la siguiente manera:

Ahora, no solamente es importante generar triángulos posicionales, sino que dentro de estos habita un secreto que permite dar profundización a la jugada para crear ocasión de gol. Este concepto es el que conocemos como la mediana del tríngulo, definida como la recta que une 
el punto medio de un lado del triángulo con el vértice opuesto, además el punto de corte de las tres medianas de un triángulo se llama baricentro, el cual juega un papel importante dentro del equilibrio de la jugada de gol. Este equilibrio se debe a que es la base de todos los posibles pases realizados en ese triángulo. (Joaquín, planeador 5, 2018).

Según lo programado, durante la implementación, se pudo observar que Joaquín se interesaba en el trabajo colaborativo a partir de las experiencias vivenciales que tenía cada uno de sus estudiantes y la manera en que las relacionaba con la geometría, buscaba que su clase fuera incluyente y motivaba la participación. El conocimiento emergía de las jugadas que se realizan en un partido de fútbol que, al ser analizadas y dialogadas, de forma crítica y rigurosa, por los estudiantes con la orientación de Joaquín llevaban a identificar los elementos de la geometría, como movimientos rectos, giros, traslaciones del balón y de los jugadores y a establecer los nuevos conceptos buscados.

Se pudo evidenciar que esta secuenciación se enmarca en una postura constructivista. El docente va orientando, generando preguntas y momentos de reflexión; brinda herramientas para que los estudiantes puedan construir su propio conocimiento. El estudiante es el protagonista, relaciona sus experiencias, hace conjeturas, socializa y reflexiona sobre su aprendizaje. En esta práctica, Joaquín tuvo libertad para plantear sus clases, y en coherencia con lo que quiere ser como docente logra proponer un contexto no rutinario, motivar a sus estudiantes a aprender y que no tengan ningún temor hacia las matemáticas. Su práctica refleja una postura constructivista, de manera clara, incluso más de lo reportado en los instrumentos cerrados.

\subsection{El caso Margarita}

Margarita quiere ser profesora de matemáticas porque considera que tiene habilidades que le permiten aprenderla de forma rápida y que, al estudiar matemáticas, no debe leer tanto. Ella espera que sus estudiantes aprendan técnicas y algoritmos para solucionar ejercicios y problemas

Que aprendan a analizar, y aprender conceptos para poder llevar a cabo procedimientos, para realizar un problema (Entrevista No 1, Margarita, 2018).

\subsubsection{Creencias epistemológicas acerca de la matemática}

A partir de los instrumentos cerrados, Margarita no tiene una postura definida sobre sus creencias acerca de la matemática. En la primera aplicación $C M 1=0,54$, prácticamente no 
tuvo cambios entre el inicio y el final del semestre, con $C M 2=0,53$. Para Margarita la matemática está en continua evolución, la tecnología es una muestra del avance y la evolución de las matemáticas, es un producto tangible del crecimiento de esta disciplina, además, de los descubrimientos que realizan los matemáticos:

[ ] Se ha visto el proceso de la evolución de las matemáticas, viendo los cambios por la historia de las matemáticas, analizando, cómo pensaban antes, los matemáticos, los filósofos, los que creaban todas esas fórmulas para hacer los procesos, ahoritica son más fáciles para comprender, entonces para mi si han evolucionado (Entrevista No 2, Margarita, 2018).

Margarita considera, además, que esa continua evolución de las matemáticas hace que no se pueda tener certeza total sobre el conocimiento matemático

Porque, así como ha evolucionado, va a evolucionar más, siempre han salido más cosas, entonces por eso nosotros como docentes tenemos que prepararnos y seguir preparándonos, para ir evolucionando a medida que eso va creciendo, porque es que eso cambia, eso cambia eso no se va a quedar así (Entrevista No 2, Margarita, 2018).

Para Margarita la matemática es una creación de la mente humana, porque es gracias al hombre que existe como disciplina, es quien la ha descubierto y, de esta forma, se ha generado un mayor conocimiento. Se nota que Margarita tiene gran gusto en lo que estudia:

La matemática es una materia, una ciencia, o sea para mí es tan bonita que pienso que adquirir el conocimiento de esta ciencia es muy, muy importante para la vida. No solamente por el conocimiento, sino para todo, porque la matemática lo tiene todo, abarca todas las ciencias (Entrevista No 1, Margarita, 2018).

Margarita también considera que las matemáticas se construyen a partir de conjeturas, de ideas que surgen y de la necesidad de resolver problemas. Señala que los resultados a los problemas matemáticos no son siempre predecibles, aunque los asocia en realidad a no tener certeza sobre los procedimientos utilizados

Porque uno hasta que haga el proceso, el procedimiento, al llegar a una respuesta no puede decir si está bien o está mal. Entonces para mí no es predecible (Entrevista No 2, Margarita, 2018).

Las anteriores afirmaciones, ubicarían a Margarita en una postura falibilista de las matemáticas, al considerar que son una creación de la mente humana, que están en continua evolución y sobre lo cual no se puede tener certeza total. Sin embargo, en algunos de los argumentos descritos se identifican varios rasgos de una postura contraria, y en otros, que se describen a continuación, se ve una inclinación absolutista, lo cual concuerda con que en el instrumento no quede en una postura claramente definida.

Margarita considera que la matemática es una ciencia formal y exacta, no hay lugar a la equivocación, las respuestas son correctas o incorrectas y su validez es comprobable

Todo se demuestra plenamente, y tiene sus bases bien formadas, sí, tiene sus fórmulas, sus 
procedimientos, su análisis, todo para llegar a un todo. Para mí la matemática es exacta (Entrevista No 1, Margarita, 2018).

Para ella, el fundamento de la exactitud de una disciplina es la capacidad que tiene de demostrar sus teorías y los procedimientos establecidos. Sin embargo, también lo asocia con el trabajo como docente, señalando la importancia que tiene para ella establecer si lo hecho por sus estudiantes, procedimentalmente, es o no correcto

Porque se hacen procesos, procedimientos, donde se llega a una respuesta final. Sí, donde yo puedo decir, califico: está bien o está mal. Verifico procedimiento, califico procedimiento y miro por qué no le dio la respuesta. Sí, eso no quiere decir que la persona éste haciendo mal las cosas, sino que de pronto tuvo mal algo, se le pasó, para llegar a una respuesta. Pero la matemática para mí es exacta por esa razón (Entrevista No 2, Margarita, 2018).

Para Margarita la demostración hace que el conocimiento matemático sea objetivo e incuestionable y considera que la matemática es un conjunto de reglas y procedimientos

Para mí es objetivo porque es algo que se puede probar, por eso para mí la matemática es, es objetiva (...) Es incuestionable y objetivo porque se puede probar (Entrevista No 1, Margarita, 2018).

Aunque argumentó que la matemática está en continua evolución, dice que ya todo está descubierto

La matemática está plenamente descubierta, para mí, que hay muchas cosas que faltan por conocer, por aprender y por descubrir, sí, pero la matemática esta descubierta (Entrevista $\mathrm{N}^{\mathrm{o}} 1$, Margarita, 2018).

Para Margarita no es posible que cualquier persona pueda crear matemáticas, nuevamente argumenta con base en la necesidad de tener conocimiento y gusto por las matemáticas

Pienso que no cualquier persona, porque una persona que no sepa, yo pienso que una persona que, que sea psicóloga, ella más que todo se prepara es metido en la mente de la persona, sí, pero ya con números no se va a meter. O sea hay personas que no les gustan los números y que escogen carreras donde solo les toque leer, no cualquier persona puede llegar a hacer eso. Pienso que no (Entrevista No 2, Margarita, 2018).

Como se observa, Margarita no tiene una postura epistemológica definida. Se ubica en el falibilismo en cuanto a los procesos de construcción histórica de la matemática y a las formas de enfrentarse a ella. Simultáneamente, se ubica en el absolutismo ya que la considera una ciencia exacta, incuestionable, objetiva, porque a partir de la demostración se evidencia su validez.

\subsubsection{Creencias sobre la enseñanza y aprendizaje}

Margarita, en ambas aplicaciones, evidenció una leve inclinación hacia el 
constructivismo con $C E A M 1=067=C E A M 2$. Para ella, las matemáticas pueden ser aprendidas aun cuando no se nazca con esa habilidad. La creatividad es un factor importante en el proceso de enseñanza y aprendizaje, y la interacción estudiante-docente facilita la construcción del conocimiento. Además, el error es una posibilidad en el desarrollo del pensamiento matemático.

Para Margarita, el profesor no es el responsable de transmitir el conocimiento ni debe tener todas las respuestas a las preguntas de los estudiantes. Por el contrario, tiene derecho a equivocarse y aprender de los demás:

Pienso que no siempre, uno también tiene fallas como profesor, a veces. Que si debe de pronto, no pasar por, o sea por no demostrar que no lo sabe, sino, de pronto sí, resolver la duda, pero bien. Pero no siempre el profesor tiene que sabérselas todas (Entrevista No 1, Margarita, 2018).

Para Margarita, la memorización de conceptos y la repetición de procedimientos no son fundamentales para el aprendizaje de las matemáticas. Lo que debe importar son los procesos que desarrolla el estudiante para resolver un problema y cómo hace uso del conocimiento que ya posee

Uno no tiene que memorizar, uno tiene que aprender a analizar, saber qué procedimiento hay que llevar, porque si uno mecaniza, las cosas pueden cambiar, llegar a un mismo resultado por diferentes maneras, entonces el memorizar es, uno se memoriza un solo camino, solo va a poder hacerlo por ese lado. En cambio si uno analiza, puede resolverlo de ambas maneras (Entrevista No 1, Margarita, 2018).

Margarita recurre a su experiencia personal para argumentar la importancia de trabajar el error como una estrategia que conlleva al aprendizaje de la disciplina

Porque uno aprende de errores, uno cuando llega a errores, eso es preciso que se le va a quedar y no se va a equivocar en lo mismo, entonces eso ha pasado con los estudiantes, hasta a mi me ha pasado, que uno comete un error y eso nunca se va a olvidar, y se le va a quedar, uno aprende de los errores (Entrevista No 1, Margarita, 2018).

En coherencia con lo anterior, Margarita considera que no es necesario que los problemas tengan una respuesta exacta, los estudiantes pueden explorar diferentes vías de solución

Yo pienso que hay muchos procedimientos para llegar a una respuesta, de pronto el estudiante toma una vía buena, pero resulta que la respuesta no le dio (Entrevista No 1, Margarita, 2018).

Una de las afirmaciones en las que Margarita cambió entre la primera y la segunda aplicación, y que disminuyó levemente su postura constructivista, es su creencia de que para aprender matemáticas se requieren habilidades especiales. Sin embargo, en su argumento se ve nuevamente el énfasis que hace en el gusto que se debe tener para poder entender, dice que es lo que ha podido observar desde su experiencia docente

Pienso que, primero que todo la matemática es algo, o sea, es una ciencia tan exacta, llena de 
procedimientos, pienso que va en el gusto de la persona, si la persona no la entiende y no le gusta, no lo va a resolver con facilidad, ni lo va a intentar, entonces pues de lo que yo he visto, de lo que tengo de experiencia como docente, a los niños se les ve eso, a los que les gusta se les facilita y a los que no, no. Pues entonces no hacen ni el mayor, ni el más mínimo intento en aprender, en entender, se les dificulta mucho (Entrevista No 2, Margarita, 2018).

Si bien señala una postura de tipo constructivista, destaca en sus argumentos aspectos fuertemente relacionados con el tradicionalismo, como la importancia que los estudiantes vean la matemática como una ciencia formal y exacta, y que, aunque hay diferentes vías para resolver problemas, siempre se llega a una respuesta exacta.

\subsubsection{Creencias y relación con la práctica}

Margarita, antes de iniciar sus prácticas, había trabajado unos meses en una institución de carácter privado. Las dos prácticas las hizo en instituciones de carácter oficial. La primera, con población adulta en jornada de fines de semana, y la segunda, en grado séptimo en un colegio femenino. Los temas que abordó en su segunda práctica estuvieron relacionados con los números enteros y sus operaciones. La codificación realizada a los planes de clase muestra que $C E A M 3=0,3$, lo que refleja una postura definida hacia el tradicionalismo. En la Tabla 2 se presentan en detalle los resultados para cada categoría y subcategoría establecidas.

Tabla 2 - Resultados codificación planes de clase de Margarita

\begin{tabular}{|l|l|r|}
\hline \multicolumn{1}{|c|}{ Categoría } & \multicolumn{1}{|c|}{ Subcategoría } & Porcentaje \\
\hline \multirow{4}{*}{ Constructivismo } & Aprendizaje & 0,00 \\
\cline { 2 - 3 } & Enseñanza - clase & 13,6 \\
& & 4 \\
\cline { 2 - 3 } & Enseñanza - docente & 6,82 \\
\cline { 2 - 3 } & Enseñanza - estudiante & 0,00 \\
\cline { 2 - 3 } & Evaluación & 11,3 \\
& & 6 \\
\hline \multirow{4}{*}{ Tradicionalismo } & Aprendizaje & 6,82 \\
\cline { 2 - 3 } & Enseñanza - clase & 25,0 \\
& & 0 \\
\cline { 2 - 3 } & Enseñanza - docente & 25,0 \\
& & 4,55 \\
\cline { 2 - 3 } & Enseñanza - estudiante & 6,82 \\
\cline { 2 - 3 } & Evaluación & 0 \\
\hline
\end{tabular}

Fuente: elaboración propia

Margarita sigue la estructura establecida para los planes de clase. En el inicio, describe que la docente saluda a las estudiantes y cuando estén organizadas les indica el tema a trabajar, por ejemplo multiplicación de enteros. Esto muestra al docente como figura de autoridad. En la conceptualización, explica el tema de manera tradicional, por ejemplo, para la multiplicación de enteros, como se observa en la Figura 3. 


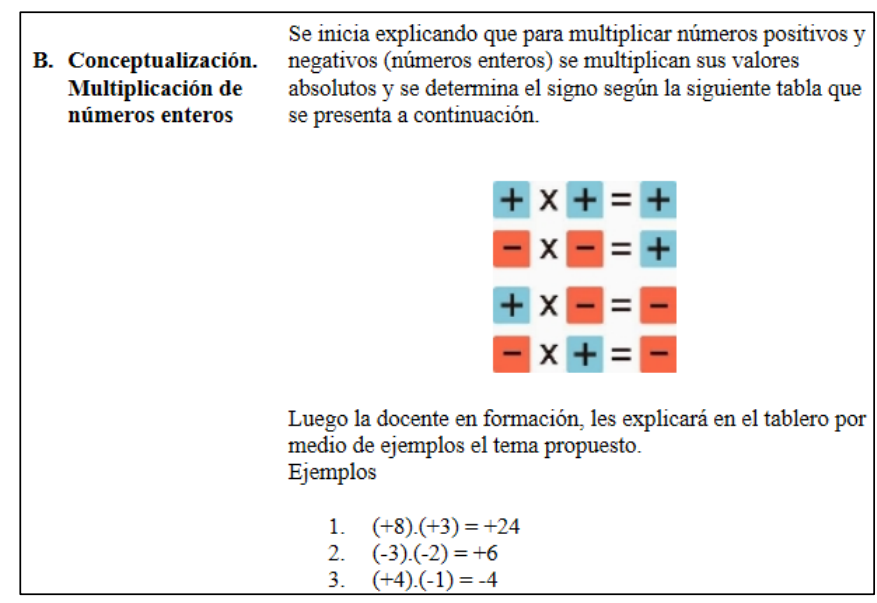

Figura 3 - Margarita, plan de clase 3

Fuente: planeador Margarita

Margarita presenta a los estudiantes reglas, sin ninguna contextualización, se dan varios ejemplos repetitivos y luego los estudiantes deben replicarlos. Se muestra al docente como el responsable de trasmitir el conocimiento, y el estudiante como quien recibe, memoriza y repite. Margarita dice, en el plan de clase, que parte de los criterios de evaluación es la participación de las estudiantes, pero también señala que una vez explicado el tema revisará en el cuaderno que las estudiantes hagan bien los ejercicios propuestos. Durante los acompañamientos se pudo observar que Margarita explicaba el tema, pedía que tomaran apuntes en el cuaderno, luego hacía seguimiento en los puestos de las estudiantes, controlando que las demás estuvieran trabajando en orden y silencio. Algunas veces pasaba luego a socializar a las estudiantes en el tablero, buscando que se llegará a la respuesta correcta.

Al cierre, presentaba aspectos relacionados con la evaluación, como entregar resueltos los ejercicios propuestos o hacer una evaluación corta. Por ejemplo, en el mismo plan de clase descrito, propone dos ejercicios rutinarios, para repetir lo hecho en clase. En este caso, la evaluación es de manera individual y presenta ejercicios diferentes para cada fila de estudiantes. Se refleja desconfianza, y esta es una manera de evitar que entre ellos puedan copiarse.

En general, se pudo observar que las clases se realizaban según lo propuesto. Margarita se mostraba muy seria con las estudiantes, hacia constantemente llamados de atención para garantizar que no hubiera ruido, que todas la escucharan con atención y, luego, pudieran repetir los procedimientos explicados. Solo en algunos planes de clase, hacia el final, hizo propuestas de incorporar solución de problemas. Se identifica claramente una enseñanza de corte tradicional, contraria a la postura señalada en el instrumento cerrado sobre sus creencias acerca de la enseñanza y el aprendizaje y argumentada, en parte, durante las entrevistas. Lo que propone para cada clase es la repetición de algoritmos, alejados de contextos reales o semi reales, con base en explicaciones iniciales dadas por ella. Se ve que el rol del docente es 
transmitir el conocimiento y controlar que las estudiantes aprendan, lo cual se verifica si pueden replicar los procedimientos y llegar a la respuesta correcta en cada ejercicio.

\section{Discusión y conclusiones}

Tanto Joaquín como Margarita mostraron, a partir de los instrumentos cerrados, que no tienen una postura claramente definida sobre las matemáticas. Poseen creencias tanto falibilistas como absolutistas sobre las matemáticas, si bien, tienen inclinación más a una de estas, lo que concuerda con otros estudios (AMIRALI; HALAI, 2010; BESWICK, 2012). Font y Ramos (2005) muestran que docentes en ejercicio no tienen creencias claras sobre la naturaleza de las matemáticas y que incorporan diferentes aspectos del platonismo y del formalismo. Sobre la enseñanza y aprendizaje, a partir de los instrumentos cerrados, ambos se inclinaron hacia el constructivismo. Yang et al. (2020) evidenciaron que los profesores en formación tienden a tener creencias mixtas sobre la naturaleza de las matemáticas y una fuerte tendencia a mantener una visión constructivista en lugar de una tradicional.

Es de resaltar que Joaquín lo hace de manera más definida al finalizar el semestre, y que tiene una práctica coherente con sus creencias y su idea de ser docente. Por su parte, Margarita evidencia a través de los planes de clase una mirada tradicional sobre el proceso de enseñanza y aprendizaje de las matemáticas. Esto se puede evidenciar en la Figura 4.

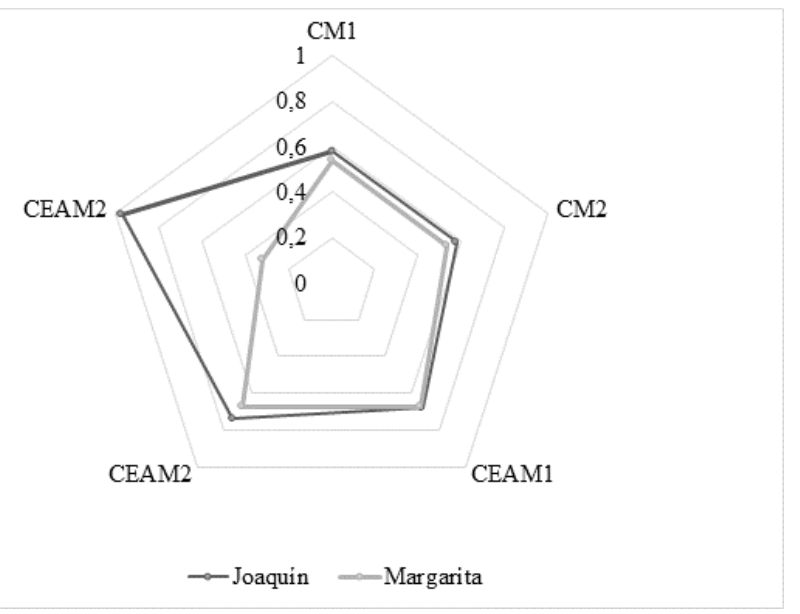

Figura 4 - Creencias reportadas por Joaquín y Margarita Fuente: elaboración propia

Los casos de Joaquín y Margarita proporcionan evidencia que permite inferir que los docentes en formación pueden tener diferentes puntos de vista sobre la matemática como disciplina y la matemática escolar, aunque no los manifiesten de manera explícita, sí queda en evidencia al observar sus prácticas. Esto también se encontró en el estudio realizado por Vesga y de Losada (2018) con docentes en ejercicio con muchos años de experiencia, quienes 
establecen una clara diferencia entre la matemática como disciplina científica y la matemática escolar.

La práctica realizada por Joaquín es coherente con su postura sobre la enseñanza y aprendizaje de las matemáticas, privilegia la construcción de conocimiento por parte de los estudiantes, promueve el aprendizaje colaborativo y utiliza contextos que permiten ver la aplicación de la matemática. De ese modo, es coherente con su idea de hacer que los estudiantes se sientan motivados por aprender y que hacerlo sea una experiencia positiva. En contraste, Margarita, aunque argumenta de manera insistente en su entrevista la importancia de que los estudiantes analicen y puedan reconstruir el conocimiento en lugar de aprender de memoria y repetir, es esto último lo que privilegia en su práctica. Sin embargo, es coherente con una parte de su idea inicial sobre qué espera de los estudiantes. En relación con la aplicación de técnicas y procedimientos, Margarita presenta la matemática como una teoría acabada, con énfasis en el dominio de técnicas y algoritmos que se derivan de la teoría (FONT, 2003).

Es posible que exista un conflicto entre las creencias tradicionales de los docentes o docentes en formación y lo que se propone en los documentos curriculares. Esto se muestra especialmente en el caso de Margarita, quien parece tener claro que lo que debería hacerse en el aula se corresponde con una postura constructivista de las matemáticas, pero no logra llevar eso a su práctica docente, o como lo señala Martínez (2018) no hay coherencia entre los enfoques propuestos de manera oficial y lo que se hace en el aula. Varios estudios han mostrado que no existe necesariamente coherencia entre las creencias reportadas y el trabajo en aula (COONEY; SHEALY; ARVOLD, 1998; PENN, 2012; VESGA; FALK, 2016).

Estos hallazgos, si bien no se pueden generalizar, son solo casos de un grupo más amplio, sí coinciden con otros estudios y plantea retos a los actuales programas de formación. Es posible diseñar y probar diferentes estrategias encaminadas a ayudar a los futuros docentes a formar y consolidar creencias enmarcadas en los desarrollos contemporáneos de la disciplina, para que esto, a su vez, les ayude al desarrollo de competencias matemáticas de niños y jóvenes (XENOFONTOS, 2018).

Es necesario, como lo plantean (COONEY; SHEALY; ARVOLD, 1998; CROOS, 2009; VESGA; DE LOSADA, 2018) que tanto programas de formación inicial como continua promuevan, de manera permanente, espacios de aprendizaje que ayuden a los docentes a dudar, reflexionar, a cuestionarse y, especialmente, a ser adaptativos, en los cuales puedan interactuar con pares y expertos y compartir sus ideas y experiencias (BOBIS et al., 2016). Examinar las creencias y conocer más sobre sus estructuras puede ser un componente clave para diseñar experiencias apropiadas para docentes en formación o en servicio (CONNER; GÓMEZ, 2019). 
También es necesario, como lo señalan Yang et al. (2020), que las clases que reciben los docentes en formación reflejen y modelen, explícitamente, creencias sofisticadas, desde el inicio del programa. En este sentido, Martínez, Araya y Berger (2017) consideran que se pueden tener resultados si se elaboran programas de desarrollo docente con énfasis en el contenido disciplinar y la relación de este con la práctica profesional, que sean sostenidos en el tiempo. Por otra parte, Hart (2002) considera que la autorreflexión puede ser un método poderoso, es necesario que el docente sea consciente de sus acciones. Nuevas investigaciones pueden realizarse desde estas perspectivas.

\section{Referencias}

ALBANESE, V.; PERALES, F. Mathematics Conceptions by Teachers from an Ethnomathematical Perspective. Bolema: Bolema: Boletim de Educação Matemática, Rio Claro, v. 34, n. 66, p.1-21, 2020. DOI: https://doi.org/10.1590/1980-4415v34n66a01.

ANDREWS, P.; XENOFONTOS, C. Analysing the relationship between the problem-solving-related beliefs, competence and teaching of three Cypriot primary teachers. Journal of Mathematics Teacher Education, Hidelberg, v. 18, n. 4, p. 299-325, 2015.

AMIRALI, M.; HALAI, A. Teachers' knowledge about the nature of mathematics: A survey of secondary school teachers in karachi, Pakistan. Bulletin of Education and Research, Dhaka, v. 32, n. 2, p. 45-61, 2010.

BALL, D.; BASS, H. Making believe: The collective construction of public mathematical knowledge in the elementary classroom. In: PHILLIPS, D. C. (Ed.). Constructivism in education. Chicago: The University of Chicago Press, 2000. p. 193-226.

BERK, D.; CAI, J. Mathematics teacher beliefs. In: PETERS, M. A. (Ed.). Encyclopedia of Teacher Education. Singapore: Springer, 2019. DOI: https://doi.org/10.1007/978-981-13-1179-6_236-1

BESWICK, K. Teachers' beliefs about school mathematics and mathematicians' mathematics and their relationship to practice. Educational Studies in Mathematics, London, v. 79, n. 1, p. 127-147, 2012. DOI: https://doi.org/10.1007/s10649-011-9333-2.

BOBIS, J.; WAY, J.; ANDERSON, J.; MARTIN, A. J. Challenging teacher beliefs about student engagement in mathematics. Journal of Mathematics Teacher Education, Hidelberg, v. 19, n. 1, p. 33-55, 2016. DOI: https://doi.org/10.1007/s10857-015-9300-4.

BUSTOS, C. E. Creencias docentes y uso de Nuevas Tecnologías de la Información y Comunicación en profesores de cinco establecimientos chilenos de educación básica y media. Universitas Psychologica, Bogotá, v. 11, n. 2, p. 511-521, 2012.

CHEN, Q.; LEUNG, F. A comparative study of teachers' mathematics beliefs in the context of curriculum reform in Hong Kong and Chongqing. The mathematics Educator, Georgia, v. 15, n. 1, p. 46-65, 2010.

CHRYSOSTOMOU, M.; PHILIPPOU, G. N. Teachers' epistemological beliefs and efficacy beliefs about mathematics. Procedia Social and Behavioral Sciences, Edinburgh, v. 9, p. 1509-1515, 2010. 
CONNER, A.; EDENFIELD, K.; GLEASON, B.; ERSOZ, F. Impact of a content and methods course sequence on prospective secondary mathematics teachers' beliefs. Journal of Mathematics Teacher Education, Hidelberg, v. 14, n. 6, p. 483-504, 2011. DOI10.1007/s10857-011-9186-8.

CONNER, A.; GOMEZ, C. Belief structure as explanation for resistance to change: The case of Robin. Journal of Mathematical Behavior, Estados Unidos de América, v. 53, p. 196-209, 2019. DOI: https://doi.org/10.1016/j.jmathb.2018.08.003.

CONTRERAS, S. A. Creencias curriculares y creencias de actuación curricular de los profesores de ciencias chilenos. Revista Electrónica de Enseñanza de las Ciencias, Vigo, v. 8, n. 2, 505-526, 2009.

COONEY, T.; SHEALY, B.; ARVOLD, B. Conceptualizing Belief Structures of Preservice Secondary Mathematics Teachers. Journal for Research in Mathematics Education, Reston, v. 29, n. 3, p. 306333, 1998.

CROSS, D. Alignment, cohesion, and change: Examining mathematics teachers' belief structures and their influence on instructional practices. Journal of Mathematics Teacher Education, Hidelberg, v. 12, n. 5, p. 325-346, 2009. DOI 10.1007/s10857-009-9120-5.

CROSS, D. Dispelling the notion of inconsistencies in teachers' mathematics beliefs and practices: A 3-year case study. Journal of Mathematics Teacher Education, Hidelberg, v. 18, n. 2, p. 173-201, 2015. DOI 10.1007/s10857-014-9276-5.

DAVIS, P.; HERSH, R.; MARCHISOTTO, E. The mathematical experience. Boston: Birkhäuser, 2012.

ERNEST, P. The impact of beliefs on the teaching of mathematics. En: KEITEL, C.; P. DAMEROW, P.; BISHOP, A.; GERDES, P. (Eds.). Mathematics, education, and society. Paris: UNESCO, 1989. p. 99-101.

ERNEST, P. Philosophy of mathematics education. Nueva York: Falmer, 1991.

ERNEST, P. Social constructivism as a philosophy of mathematics. Albany: State University of New York Press, 1998.

ERNEST, P. Forms of knowledge in mathematics and mathematics education: Philosophical and rhetorical perspectives. Educational Studies in Mathematics, London, v. 38, p. 67-83, 1999.

FONT, V. Matemáticas y cosas. Una mirada desde la Educación Matemática. Boletín de la Asociación Matemática Venezolana, v. 10, n. 2 , p. 249-279, 2003.

FONT, V.; RAMOS, A. Objetos personales matemáticos y didácticos del profesorado y cambio institucional. El caso de la contextualización de funciones en una facultad de ciencias económicas y sociales. Revista de Educación, Madrid, n. 338, p. 309-346, 2005.

HANDAL, B.; HERRINGTON, A. Mathematics teachers' beliefs and curriculum reform. Mathematics Education Research Journal, Australia, v. 15, n. 1, p. 59-69, 2003.

HART, L. A four year follow-up study of teachers' beliefs after participating in a teacher enhancement project. In: LEDER, G. C.; PEHKONEN, E.; TO“RNER, G. (Eds.). Beliefs: A hidden variable in mathematics education? Dordrecht: Kluwer Academic Publishers. 2002. p. 161-176.

HOFER, B.; PINTRICH, P. The development of epistemological theories: Beliefs about knowledge and knowing and their relation to learning. Review of Educational Research, California, v. 67, n. 1, p. 88-140, 1997. 
LAKATOS, I. A renaissance of empiricism in the recent philosophy of mathematics. The British Journal for the Philosophy of Science, Oxford, v. 27, n. 3, p. 201-223, 1976.

LEINO, J. Theoretical considerations on constructivism. In: AHTEE, M.; PEHKONEN, E. (Eds.). Constructivist viewpoints for school teaching and learning in mathematics and science. Helsinki: Universidad de Helsinki, 1994. p. 13-18.

LERMAN, S. Alternative perspective of the nature of mathematics. British Educational Research Journal, Routledge, v. 16, p. 53-61, 1990.

MARTÍNEZ, F. ¿Por qué es tan difícil mejorar los niveles de aprendizaje? A propósito de las nuevas reformas a la educación básica Mexicana. Perfiles Educativos, Ciudad de México, v. 11, n. 159, p. 162-176, 2018.

MARTÍNEZ, M.; ARAYA, P.; BERGER, B. Descripción del cambio del profesor de matemática desde su propia perspectiva a partir de una experiencia en torno a resolución de problemas de final abierto. Bolema: Boletim de Educação Matemática, Rio Claro, v. 31, n. 59, p. 984-1004, 2017. DOI: https://dx.doi.org/10.1590/1980-4415v31n59a07.

MANSOUR, N. Science Teachers' Beliefs and Practices: Issues, Implications and Research Agenda. International Journal of Environmental and Science Education, Turkey, v. 4, n. 1, p. 25 48, 2009.

MCMINN, M.; ALDRIDGE, J.; HENDERSON, D. Learning environment, self-efficacy for teaching mathematics, and beliefs about mathematics. Learning Environ Res, 2020. Disponible en: https://ezproxy.uan.edu.co:2072/10.1007/s10984-020-09326-x. Acceso en: 26.09.2020.

PAJARES, M. F. Teachers' beliefs and educational research: Cleaning up a messy construct. Review of Educational Research, California, v. 62, n. 3, p. 307-333, 1992.

PANTZIARA, M.; KARAMANOU, M.; PHILIPPOU, G. Teachers'beliefs and knowledge related to the Cyprus mathematics curriculum reform. En: UBUZ, B.; HASER, Ç.; MARIOTTI, M. A. (Eds.). Proceedings of the Eighth Congress of European Research in Mathematics Education. Ankara: Middle East Technical University, 2013. p. 1399 - 1408.

PEHKONEN, E.; TÖRNER, G. Introduction to the abstract book for the Oberwalfach meeting on belief research. In: PEHKONEN, E; TORNER, G. (Eds.). Proceedings of the workshop in Oberwalfach on mathematical beliefs and their impact on teaching and learning of mathematics. Oberwolfach: Gerhard Mercator Universitat Duisburg, 1999. p. 3-10.

PENN, A. The Alignment of Preservice Elementary School Teachers' Beliefs concerning Mathematics and Mathematics Teaching. 2012. Disertación (Master of education) - Queen's University, Kingston, 2012.

PHILLIPS, D. C. An opinionated account of the constructivist landscape. En: PHILLIPS, D. C. (Ed.). Constructivism in Education. Chicago: The University of Chicago Press, 2000. p. 1-18.

PONCE, R. Las creencias sobre los factores que generan un ambiente exitoso de aprendizaje en profesores de educación superior. Revista Iberoamerica de Educación, Ciudad de Mexico, v. 7, n. 46, p. 1-12, 2008. DOI: https://doi.org/10.35362/rie4671912.

RAHIME, C. A Review of Research in Turkey on Beliefs about Teaching. Journal of Education, Whashington D.C., v. 30, n. 3, p. 48-59, 2015.

ROCCO, T.; BLISS, L.; GALLAGHER, S; PÉREZ, A. Taking the Next Step: Mixed Methods 
Research in Organizacional Systems. Information Technology, Learning, and Performance Journal, Waynesville, v. 21, n.1, p. 19-29, 2003.

SKOTT, J. The forced autonomy of mathematics teachers. Educational Studies in Mathematics, London, v. 55, n. 1-3, p. 227-257, 2004.

DOI: $10.1023 /$ B:EDUC.0000017670.35680.88.

SCHOMMER, M. Effect of beliefs about the nature of knowledge incomprehension. Journal of Educational Psychology, Whashington D.C., v. 82, n. 3, p. 498-504, 1990.

STRAUSS, A; CORBIN, J. Bases de la investigación cualitativa: Técnicas y procedimientos para desarrollar la Teoría Fundamentada. Medellín: Universidad de Antioquia. 2002.

STEINER, H. Philosophical and Epistemological Aspects of Mathematics and Their Interaction with Theory and Practice in Mathematics Education. Learning of Mathematics, Heidelberg, v. 7, n. 1, p. 7-13, 1987. Disponible en: http://www.jstor.org/stable/40247880. Acceso en: 01.03.2019.

SWAN, M. The impact of task-based professional development on teachers' practices and beliefs: A design research study. Journal of Mathematics Teachers Education, Hidelberg, v. 10, p. 217-237, 2007. DOI: https://doi.org/10.1007/s10857-007-9038-8.

THOMPSON, A. The relationship of teachers'conceptions of mathematics teaching to instructional practice. Educational Studies in Mathematics, London, v. 15, p. 105-127, 1984.

VESGA, G; FALK, M. Creencias epistemológicas de docentes de matemáticas acerca de la matemática, su enseñanza y su relación con la práctica docente. Revista Papeles, Bogotá, v. 8, n.16, p. 11-25. Disponible en http://revistas.uan.edu.co/index.php/papeles/article/view/449. 2016. Acceso en: 05042019

VESGA, G; ANGEL, Z. Impacto del diseño de Microclaes sobre las creencias epistemolóticas de las matemáticas, su enseñanza y aprendizaje de docentes en formación. En: Actas del Congreso Iberoamericano de Docentes. Asociación Formación IB. 2018. Recuperado de http://formacionib.org/congreso/533.pdf. Acceso en: 04.03.2019.

VESGA, G; DE LOSADA, M. Creencias epistemológicas de docentes de matemáticas en formación y en ejercicio sobre las matemáticas, su enseñanza y aprendizaje. Revista Colombiana de Educación. Bogotá, v. 74, p. 243-267. 2018. DOI: https://doi.org/10.17227/rce.num74-6909

WHITE-FREDETTE, K. What is mathematics? An exploration of teachers' philosophies of mathematics during a time of curriculum reform. Dissertation, Georgia State University, 2009. Disponible en: https://scholarworks.gsu.edu/msit_diss/46 Acceso en: 04.05.2017.

XENOFONTOS, C. Greek-Cypriot elementary teachers' epistemological beliefs about mathematics. Teaching and Teacher Education, London, v. 70, p. 47-57, 2018. DOI: https://doi.org/10.1016/j.tate.2017.11.007.

XIE, S.; CAI, J. Teachers' Beliefs about Mathematics, Learning, Teaching, Students, and Teachers: Perspectives from Chinese High School In-Service Mathematics Teachers. International Journal of Science and Mathematics Education, California, v. 19, p. 747-769, 2021. Disponible en: https://ezproxy.uan.edu.co:2072/10.1007/s10763-020-10074-w. Acceso en: 05.03.2019.

YANG, X; KAISER, G; KÖNING, J; BLÖMEKE, S. Relationship between pre-service mathematics teachers' knowledge, beliefs and instructional practices in China. ZDM Mathematics Education, Berlín, v. 52, p. 281-294. 2020. Disponible en: https://ezproxy.uan.edu.co:2072/10.1007/s11858-02001145-x. Acceso en: 05.03.2020. 
YIN, R. Case study research: Design and methods. 4. ed. Thousand Oaks: Sage, 2008.

ZHAO, W.; MOK, I. A. C.; CAO, Y. Curriculum reform in china: student participation in classrooms using a reformed instructional model. International Journal of Educational Research, Amsterdam, v. 75, p. 88 -101, 2016. DOI: https://doi.org/10.1016/j.ijer.2015.10.005.

Submetido em 02 de Julho de 2019. Aprovado em 24 de Novembro de 2020. 